\title{
Improved Differentiation of Mesenchymal Stem Cells into Hepatocyte-like Cells using FGF4 and IGF-1 in 3D Culture
}

\author{
Khodabandeh $Z^{1}$, Vojdani $Z^{2}$, Jaberipour $\mathrm{M}^{3}$, Hosseini $\mathrm{A}^{4}$, Bahmanpour $\mathrm{S}^{5}$, Talaei-Khozani $\mathrm{T}^{6^{*}}$
}

Research Article

\author{
${ }^{1}$ Assistant Professor, Laboratory for Stem Cell Research, Anatomy Department, Transgenic Technology Research Center, Shiraz University of Medical \\ Sciences, Shiraz, Iran. \\ ${ }^{2}$ Associated Professor, Laboratory for Stem Cell Research, Anatomy Department, Shiraz University of Medical Sciences, Shiraz, Iran. \\ ${ }^{3}$ Assistant Professor, Institute for Cancer Research, Shiraz University of Medical Sciences, Shiraz, Iran. \\ ${ }^{4}$ Institute for Cancer Research, Shiraz University of Medical Sciences, Shiraz, Iran. \\ ${ }^{5}$ Professor, Laboratory for Stem Cell Research, Anatomy Department, Shiraz University of Medical Sciences, Shiraz, Iran. \\ ${ }^{6}$ Associated Professor, Tissue Engineering Lab, Tissue Engineering Department, Advance School of Medical Sciences and Technology, Shiraz Univer- \\ sity of Medical Sciences; Laboratory for Stem Cell Research, Anatomy Department, Shiraz University of Medical Sciences, Shiraz, Iran.
}

\begin{abstract}
Human Umbilical Cord Mesenchymal Stem Cells (UCMSCs) are considered as an excellent candidate for cell therapy to treat end-stage liver disease. Fibroblast Growth Factor-4 (FGF4), Hepatocyte Growth Factor, and Insulin-like Growth Factor-1 are some of the critical cytokines involved in liver development and regeneration. To evaluate the differentiation potency of cells into hepatocyte-like cells we used these cytokines. UCMSCs were isolated from Wharton's jelly of fullterm infants. The cells were characterized as MSCs by flow-cytometry and their multilineage differentiation capacity. Then, UCMSCs were cultured in 3D collagen scaffold and hepatogenic media with or without FGF4 for 21 days and the data were compared to control. The expression of liver specific genes was evaluated by real-time quantitative RT-PCR and immunocytochemistry.

These cells expressed MSC markers and could differentiate into adipocytes and osteocytes. A non-significant higher level of liver specific genes, such as cytokeratin-18 and 19, alpha-fetoprotein and albumin, and also a significant higher level of CYP2B6 expressed by UCMSCs in hepatogenic medium containing FGF4 compared with control. In some specimens, cytokeratin-19-positive cells surrounded a luminal space within collagen scaffolds. Liver-specific marker expression was increased by pre-exposing the cells to FGF4 before treating with IGF-1 and HGF in 3D collagen scaffold.

Keywords: Umbilical Cord Mesenchymal Stem Cells; 3D Culture; Collagen Scaffold; Fibroblast Growth Factor-4; Hepatocyte-Like Cell; Insulin-Like Growth Factor-1.

Abbreviations: UCMSCs: Human Umbilical Cord Mesenchymal Stem Cells; FGF4: Fibroblast Growth Factor 4; HGF: Hepatocyte Growth Factor; IGF-1: Insulin-like Growth Factor-1; MSCs: Mesenchymal Stem Cells; ICG: Indocyanine green; PAS: periodic acid Schiff; CK-18: cytokeratin-18; CK-19: Cytokeratin-19; AFP: alpha-fetoprotein; G6P: glucose 6 phosphatase; PEPCK: phosphoenolpyruvate carboxykinase; TAT: tyrosine amino transferase; FBS: Fetal Bovine Serum; OSM: oncostatin M; RT-PCR: Reverse Transcription Polymerase Chain Reaction; PBS: Phosphate-Buffered Saline; HepPar1: Hepatocyte paraffin 1; DAB: Diaminobenzidine; CYP2B6: Cytochrome P450 2B6.
\end{abstract}

\section{*Corresponding Author:}

Tahereh Talaei-Khozani,

Associated professor, Tissue Engineering Lab, Tissue Engineering Department, Advance School of Medical Sciences and Technology, Shiraz University of Medical Sciences; Laboratory for Stem Cell Research, Anatomy Department, Shiraz University of Medical Sciences, Shiraz, Iran.

Tel: ++987112304372

Fax: ++987112304372

Email: talaeitkh@gmail.com

Received: June 07, 2015

Accepted: July 27, 2015

Published: July 28, 2015

Citation: Talaei-Khozani T et al., (2015) Improved Differentiation of Mesenchymal Stem Cells into Hepatocyte-like Cells using FGF4 and IGF-1 in 3D culture. Int J Stem Cell Res Transplant 03(3), 109-117. doi: http://dx.doi.org/10.19070/2328-3548-1500018

Copyright: Talaei-Khozani $\mathbf{T}^{\mathcal{O}} 2015$. This is an open-access article distributed under the terms of the Creative Commons Attribution License, which permits unrestricted use, distribution and reproduction in any medium, provided the original author and source are credited.

\section{Introduction}

Human Umbilical Cord Matrix Mesenchymal Stem Cells (UCMSCs) isolated from Wharton's jelly show multipotent properties. They showed higher differentiation potential compared to adult Mesenchymal Stem Cells (MSCs). These cells can express a low level of major histocompatibility complex antigens (class I and II) and have immunomodulatory properties [1]. The potential of UCMSCs to differentiate into various lineages, such as adipocytes, osteocytes, and chondrocytes, self-renewal capacity, and high proliferation rate, have been demonstrated [2]. A recent study confirmed the highest efficiency and viability of UCMSCs in the fifth and sixth passages in comparison to other sources, such as umbilical cord blood and bone marrow. UCMSCs can be considered as a non-invasive, easily available cell source and an excellent candidate for cell therapy at end stage liver disease $[1,3]$. Various in vitro differentiation protocols and subsequent cellular therapies need a sufficient source of viable stem cells with the capacity to be functional. UCMSCs have been detected to express both embryonic and adult MSC markers [4]. They can also ex- 
press several early hepatic markers, such as albumin, alpha fetoprotein (AFP), and cytokeratin-19 (CK-19), mid and late hepatic markers, such as glucose 6 phosphatase (G6P), connexin 32, and CK-18 [5, 6] and a low level of hematopoietic markers, such as ckit and phosphoenolpyruvate carboxykinase (PEPCK) [7]. Based on these investigations, UCMSCs demonstrated an intermediate phenotype between the hepatoblast and the mature hepatocyte; because, the late hepatic markers, including Cytochrome P450 2B6 (CYP2B6), Hepatocyte paraffin 1 (HepPar1), and Tyrosine Amino Transferase (TAT), have not been reported yet to be expressed by UCMSCs [8]. A lot of investigations have shown the differentiation potential of MSCs from various sources toward hepatocytes $[9,10]$. For instance, liver specific genes, such as AFP and albumin have been shown to be expressed by rat bone marrow-derived MSCs after 21 days of incubation in the hepatogenic medium. Hepatocyte Growth Factor (HGF) is the decisive factor and induces the expression of c-met, the HGF receptor, in the target cells [11]. In vitro studies have revealed that HGF induced the expression of liver specific genes (albumin, CK) in human mononuclear cord blood cell culture [12]. FGF-4 has mitogenic and angiogenic effects and can induce MSCs to proliferate and differentiate toward endoderm and subsequently hepatogenic fate [13]. Moreover, it has been demonstrated that a combination of Fibroblast Growth Factor-4 (FGF4) and HGF could induce bone marrow-derived MSCs into functional hepatocyte-like cells [14]. During the initiation step of in vitro hepatocyte differentiation, FGF4 is a necessary growth factor for induction of cells toward definitive endoderm and exerts mitogenic and angiogenic effects [15].

Most of the investigations on transdifferentiation of adult stem cells toward hepatic lineage have focused on 2D monolayer culture system. Cell-cell contact plays a critical role in 3D tissue fabrication, such as liver [6]. Culturing the stem cells in a 3D collagen scaffold mimics in vivo cytoarchitecture and provides an appropriate microenvironment for cell-cell interaction as well as cell migration and improves tissue organization [16]. The functions and stability of the hepatocyte phenotype can be improved during the differentiation in 3D multicellular aggregates compared to monolayer cultures [17].

Several studies have shown that human UCMSCs had the ability to differentiate into hepatocyte-like cells; however, the differentiated cells were not functional [18]. A previous study demonstrated that the combination of Insulin like Growth Factor-1(IGF-1) with HGF could improve the differentiation of MSCs toward hepatocytes [19]. Therefore, designing an effective, simple, and innovative method to produce functional hepatocyte-like cells seems to be necessary. The present study aims to evaluate the ability of human UCMSCs as an accessible and feasible source to differentiate toward hepatocyte-like cells by a 3D culture system in the presence of hepatogenic media containing IGF-1 with or without FGF4.

\section{Materials and Methods}

\section{Primary Culture of UCMSCs}

Human umbilical cords were obtained from newborns delivered through cesarean sections after obtaining written informed consent from their parents. They were transferred to the laboratory in cold Phosphate-Buffered Saline (PBS) containing 100U/mL penicillin, $100 \mu \mathrm{g} / \mathrm{mL}$ streptomycin (Sigma Aldrich, UK). Afterwards, the two arteries were removed completely, the umbilical vein was cut open, and the endothelia were crushed using a sterile blade. Then, the umbilical cords were cut into small pieces, about $5 \mathrm{~mm}$, and the explants were put in the culture dishes. After $15 \mathrm{~min}$, $\alpha$-MEM (Gibco BRL, life technology, Germany) containing 10\% Fetal Bovine Serum (FBS) (Gibco BRL), 1\% L-glutamine (Sigma Aldrich, UK), and $100 \mathrm{U} / \mathrm{mL}$ penicillin, $100 \mu \mathrm{g} / \mathrm{mL}$ streptomycin were added to the culture plates.

\section{Surface Marker Characterization}

The cells were harvested at the third passage and the cell suspension was adjusted at a concentration of $1 \times 10^{6}$ cells $/ \mathrm{mL}$ in cold PBS containing $10 \%$ FBS as the blocking solution for $20 \mathrm{~min}$. After that, the cells were incubated in FITC-conjugated anti-CD44 and CD144, phycoerythrin-conjugated anti-CD34 and CD106, and PerCP-conjugated anti- CD105 antibodies (all from Abcam, UK) for $30 \mathrm{~min}$. Then, the cells were washed and resuspended in PBS containing 10\% FBS. The frequencies of positive cells were evaluated by a FACS calibrated instrument (BD, USA).

\section{Osteogenic and Adipogenic Differentiation Potential of UCMSCs}

The cells were cultured in the presence of osteogenic (MACS, Germany) and adipogenic media (StemCell Technologies Inc, Canada) for 4 and 3 weeks, respectively. The media were changed twice a week. After 4 weeks, the differentiated cells in the osteogenic medium were fixed in $4 \%$ formaldehyde and stained with Alizarin red/S. The cells cultured in the presence of adipogenic differentiation medium were fixed at $96 \%$ methanol and stained by oil red. Images were captured using Nikon Eclipse TE2000-U inverted microscope (Nikon, Japan).

\section{Hepatocyte Differentiation}

Rat tail type I collagen (Gibco, A10483-01) was prepared according to the protocol with some modification just before use [26]. Collagen solution at the concentration of $3 \mathrm{mg} / \mathrm{mL}$ was reconstructed with 10X DMEM (Gibco BRL) at 8:1 ratio on ice by slow and gentle pipetting. The color of DMEM changed from red to yellow, indicating the acidic $\mathrm{pH}$ of the collagen solution. Wharton's jelly-derived MSCs were harvested at passage 4 or 5 and an aliquot of the cells at a density of $7 \times 10^{5}$ cell $/ \mathrm{mL}$ with $1 \mathrm{mg} / \mathrm{mL}$ collagen at $1: 1 \mathrm{v} / \mathrm{v}$ ratio was prepared. The cells were incubated at $37^{\circ} \mathrm{C}$ for $1 \mathrm{~h}$ to form collagen gel. Then, the culture medium containing $10 \%$ FBS was added.

In this study, the 3D cultured-cells were used in 3 different groups. The first group that was incubated in DMEM (low glucose) supplemented with $5 \% \mathrm{FBS}, 100 \mathrm{U} / \mathrm{mL}$ penicillin, $100 \mu \mathrm{g} / \mathrm{mL}$ streptomycin was considered as the control group.

The second group was cultured in hepatogenic medium containing DMEM supplemented with 5\% FBS, 20ng/mL HGF, 20ng/ mL IGF-I (both from Peprotech, UK), 100nM dexamethasone (Sigma-Aldrich, UK), 100U/mL penicillin and $100 \mu \mathrm{g} / \mathrm{mL}$ streptomycin for 7 days. Afterwards, $10 \mathrm{ng} / \mathrm{mL}$ oncostatin M (OSM) (Peprotech, UK) was also added to the hepatocyte differentiation medium for 2 weeks.

The third group was first incubated with DMEM supplemented with 5\% FBS and 10ng/mL FGF4 (Peprotech, UK) for 2 days. 
Then, the culture medium was replaced with hepatogenic medium containing DMEM supplemented with $5 \%$ FBS, $20 \mathrm{ng} / \mathrm{mL}$ HGF, 100nM dexamethasone, $100 \mu \mathrm{g} / \mathrm{mL}$ penicillin, and $100 \mathrm{U} /$ $\mathrm{mL}$ streptomycin for an additional 5 days. The differentiation protocol was continued by adding $10 \mathrm{ng} / \mathrm{mL}$ OSM (Figure 1). In all the groups, the cultures were continued for 21 days and half of the culture media was changed every 3 days.

\section{Real Time RT-PCR Analysis}

Total RNA was extracted from UCMSCs by Biazol isolation reagent (Bioflux, Japan) according to the manufacturer's instructions. The concentration and purity of the extracted RNA were determined by Biophotometer (Eppendorf, Germany). Then, total

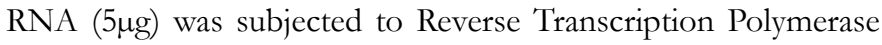
Chain Reaction (RT-PCR) to make cDNA. The cDNA $(2 \mu \mathrm{l})$ was used as the template for real-time PCR reactions. The primers were designed using Primer-BLAST online program based on the human DNA sequences found in the genebank [20] (Table 1). Real-time PCR was performed using the SYBRs Green PCR Master Mix (Applied Biosystems, USA) in 50 cycles with an initial de- naturation temperature of $95^{\circ} \mathrm{C}$ for 10 min continued by cycling denaturation in $95^{\circ} \mathrm{C}$ for 15 seconds, annealing at $60^{\circ} \mathrm{C}$ for $1 \mathrm{~min}$. After amplification, melting curve analyses were performed to ensure that no primer-dimers were produced, confirming the accuracy of the reactions. The accuracy of the reactions was checked by gel electrophoresis of PCR products. The cDNA of HepG2 cell line was used as the positive control.

Normalization of target gene expression levels was performed using $18 \mathrm{~S}$ as a housekeeping gene. Besides, the following formula was used for computation of Relative Quantification (RQ): $2^{-\Delta \Delta \mathrm{Ct}}$.

\section{Immunohistochemical Staining}

After washing with PBS, collagen gels were immersed in Bouin's fixative, embedded in paraffin, cut into $10-\mu \mathrm{m}$ thick sections, and mounted on glass slides. The sections were deparaffinized in $\mathrm{xy}$ lene (Sigma, UK), rehydrated in ethanol, and rinsed in PBS. The slides were incubated in $3 \% \mathrm{H}_{2} \mathrm{O}_{2}$ (Sigma, UK) to suppress the endogenous peroxidase activity for $20 \mathrm{~min}$. The standard heatmediated citrate buffer antigen retrieval protocol was used for

Figure 1. Schematic diagram of the protocol steps for each experimental group.
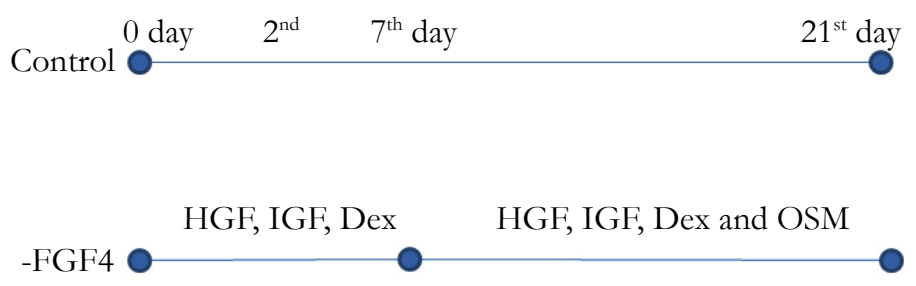

+ FGF4 FGF4 HGF, IGF, Dex HGF, IGF, Dex and OSM

Table 1. The primer sequences used for Quantities real time PCR

\begin{tabular}{|c|c|c|}
\hline Primer & sequence & Size (base pair) \\
\hline \multirow[t]{2}{*}{ Albumin } & 5'ACAGAGACTCAAGTGTGCCAGT-3' & 198bp \\
\hline & 5'-GCAAGGTCCGCCCTGTCATC-3' & \\
\hline \multirow[t]{2}{*}{ Alpha-fetoprotein } & 5'-TTCATATGCCAACAGGAGGC-3' & $152 \mathrm{bp}$ \\
\hline & 5'-TGAGAAACTCTTGCT'TCATCGT-3' & \\
\hline \multirow[t]{2}{*}{ Cytokeratin-18 } & 5'AAATCCGGGAGCACT'TGGAG-3' & $132 \mathrm{bp}$ \\
\hline & 5'-CAATCTGCAGAACGATGCGG-3 & \\
\hline \multirow[t]{2}{*}{ Cytokeratin-19 } & 5'-ACTACACGACCATCCAGGAC-3' & $126 \mathrm{bp}$ \\
\hline & 5'CCGTCTCAAACTTGGTTCGGA-3 & \\
\hline \multirow[t]{2}{*}{ CYP2B6 } & 5'-TTCTTCCGGGGATATGGTGT-3' & $91 \mathrm{bp}$ \\
\hline & 5'-TCCCGAAGTCCCTCATAGTG-3' & \\
\hline \multirow[t]{2}{*}{ Hepatic Nuclear Factor 4} & 5'AAGAAATGCT'TCCGGGCTGG-3' & 156bp \\
\hline & 5'-GACGGGGGAGGTGATCTGTC-3' & \\
\hline \multirow[t]{2}{*}{ Glucose-6-phosphatase } & 5'-CGACGAAGCGCAGACAG-3' & $113 \mathrm{bp}$ \\
\hline & 5'-GTATCCGACTGATGGAAGGC-3' & \\
\hline \multirow[t]{2}{*}{ Claudin } & 5'CTTCTTGCAGGTCTGGCTAT-3' & 196bp \\
\hline & 5'AGGTTGTT'T'T'TCGGGGACAG-3' & \\
\hline \multirow[t]{2}{*}{$18 \mathrm{~S}$} & 5'-GTTGATTAAGTCCCTGCCCT-3' & $75 \mathrm{bp}$ \\
\hline & 5'-TCCGAGGGCCTCACTAAACC-3' & \\
\hline
\end{tabular}


anti-CK18 and -AFP antibodies. The sections stained for CK19 were incubated with proteinase K (Sigma Adrich, UK). Then, the slides were washed with PBS for three times and incubated with PBS containing $10 \%$ goat serum to block non-specific binding sites for $20 \mathrm{~min}$. Then, primary anti-CK18, 19 antibodies and AFP (1:100 dilution, Abcam, UK) were added to the slides at $4^{\circ} \mathrm{C}$ overnight in a humidified dark chamber. Negative controls were performed by omitting the primary antibodies. The slides were washed in PBS 2X for 10 min. After that, they were incubated in Super Enhancer for 15 min and then in Polymer_HRP (Envision) for $30 \mathrm{~min}$. Fresh prepared-diaminobenzidine (DAB) (Sigma, UK) solution containing $\mathrm{H}_{2} \mathrm{O}_{2}$ was added to the slides for $3 \mathrm{~min}$ and $\mathrm{DAB}$ was removed by rinsing the slides in distilled water. After all, the slides were counterstained with hematoxylin, dehydrated, and mounted.

\section{Immunofluorescence}

For immunofluorescence staining of $3 \mathrm{D}$ culture cells, the slides were deparafinized in xylene, rehydrated in ethanol and rinsed in distilled water. The slides were permeabilized with $0.5 \%$ Triton X-100 (Sigma, UK) for $10 \mathrm{~min}$ and blocked with $10 \%$ goat serum in PBS for $20 \mathrm{~min}$. The sections were incubated with 1:100 dilutions of anti-albumin FITC-conjugated (Abcam, UK) at dark for 1h. After washing with PBS for 3 times, the slides were counterstained with DAPI (Sigma, UK), washed with PBS and analyzed by the fluorescence microscope (Olympus, BX51, and Japan).

\section{Periodic Acid Schiff (PAS) Assay}

The 3D culture sections were deparaffinized and oxidized in $1 \%$ periodic acid for 5 minutes. Sections were then incubated in
Schiff reagent for $15 \mathrm{~min}$, and washed in running tap water for 5-10 min. The sections were then dehydrated and mounted.

\section{Indocyanine Green Test}

The cells were exposed to $1 \mathrm{mg} / \mathrm{mL}$ Indocyanine green (ICG) (Cardiogreen; Sigma Aldrich, UK) at $5 \% \mathrm{CO}_{2}$ at $37^{\circ} \mathrm{C}$ for $1 \mathrm{~h}$. The cells were then washed with PBS for three times. After that, they were incubated with fresh William's medium containing 10\% FBS for $6 \mathrm{~h}$. The medium was collected and the concentration of ICG released from the cells was determined at an optical density of $820 \mathrm{~nm}$ by spectrophotometer analyses (Cecil 1011, UK) [21].

\section{Statistical Analysis}

All the statistical analyses were performed using GraphPad Prism 5 software (GraphPad, USA) and Mann-Whitney test. P-value $<0.05$ was considered as statistically significant.

All the experiments were performed in triplicate.

\section{Results}

\section{Characterization of UCMSCs}

Flow cytometry data showed that UCMSCs were positive for the common MSCs surface markers, such as CD90 (94.5\%), CD73 (98.5\%), CD44 (92.7\%), CD105 (77.2\%), and CD106 (43.5\%) and the reaction was negligible for hematopoietic stem cell (CD34, $1.06 \%$ ) and endothelial cell (CD144, 2.06\%) markers (Figure. 2). The osteogenic and adipogenic capacities of these cells were eval-

Figure 2. The flow cytometry showed the frequency of the umbilical cord mesenchymal stem cells which reacted to CD90, CD73, CD44, 105, and 106; however, the frequency of the cells which reacted to CD 34 and 144 was negligible.
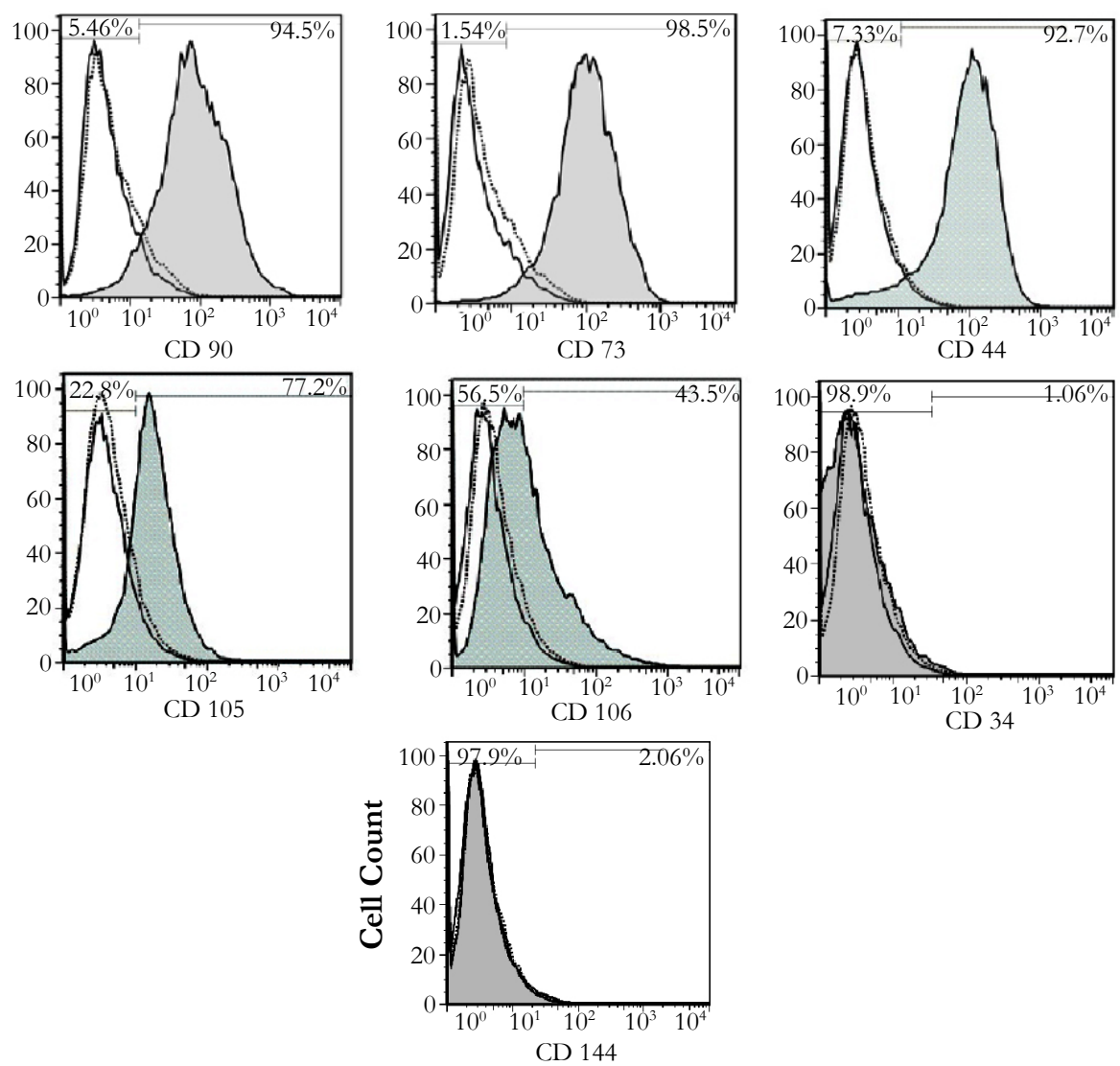
uated by alizarin red S and oil red staining, respectively (Figure. 3). These results confirmed the multipotency of the UCMSCs.

\section{Cell Morphology}

The primary cells in the conventional culture condition showed a fibroblast-like morphology which is a typical characteristic of MSCs. The morphology of UCMSCs was also spindle or star shape; however, the morphology of the cells treated with hepatogenic media was changed into a round shape after supplementation of the culture media with OSM (Figure. 4). Also, some cells had a tendency toward enclosing a luminar space in the $3 \mathrm{D}$ scaffolds. Human UCMSCs were proliferated more rapidly in response to the growth factors presented in the hepatogenic media; in such a way that the density of the cells was higher in the growth factor-treated cultures compared to the control cultures.

\section{Real Time PCR}

The expression profiles of albumin, AFP, CK18, CK19, and CYP2B6 mRNA were evaluated in the cultured cells in all the groups after 21 days. A low level of liver-specific genes was also expressed by the human UCMSCs which were cultured in a condition without any hepatogenic differentiation factors (control cultures).

Similarly, these genes were detected in the cells cultured in hepatogenic media supplemented with or without FGF4. The hepatogenic media supplemented with FGF4 induced the cells to express a non-significant higher level of these early and mid-stage hepatic markers including albumin, AFP, CK18, CK19, HNF4- $\alpha$, and a significant higher level of CYP2B6 (180 , 44 , 4 , 12.6 , 29.6 and $21000 \sim$ fold, respectively) than the control cultures. A very low level of the CYP2B6 mRNA expression was also detected in undifferentiated UCMSCs. The cells in control cultures could express more claudin compared with the cells exposed to the hepatogenic media (Figure. 5). In the cultures exposed to hepatogenic media without FGF4, albumin, HNF4- $\alpha$ and AFP were expressed 27.3 , 17 and 16 fold higher than control cultures, respectively. However, the quantity of the CK18 and 19 did not change after treatment of the cells with hepatogenic media. The expression of G6P was decreased in the cells exposed to hepatogenic factors with or without FGF4. The statistical analyses did not show any significant difference between the three groups except for CYP2B6 $(\mathrm{P}=.02)$. After gel running, the band of the PCR product was formed at the appropriate position (data not shown).

\section{Immunohistochemistry and Immunofluorescence}

Immunohistochemistry staining of the differentiated UCMSCs revealed the expression of AFP, CK-18, and CK-19 (Figure. 6) in different cultures. The expression of the early hepatogenic markers was low in 3D scaffolds in the absence of hepatogenic media after 10 days of culturing. The percentages of the positive cells for CK18, 19, and AFP are summarized in Table 2. In order to find the impacts of long term culture of the UCMSCs in 3D condition on the level of the gene expression, the current study compared the expression of the hepatocyte-specific markers in long term (21 days) with short term cultures (10 days). Although the UCMSCs' morphology did not change in the control cultures, the results showed a significant increase in the intensity of the reactions to various antibodies and besides, the frequency of the AFP-positive cells in the cells cultured within a 3D collagen scaffold for long-term (21 days) was increased compared to those cultured for a short period of time $(\mathrm{P}=0.013)$. The cells which were exposed to hepatogenic media with or without FGF4 also expressed early hepatogenic markers along with cell morphology modification. These cells showed a significant increase in CK19 and AFP expression compared to the control groups $(\mathrm{P}=0.0001$ and $\mathrm{P}=0.0001)$. Moreover, the frequency of the CK18 increased significantly after pre-exposure of the cells to FGF4 compared to the cultures treated with just hepatogenic medium $(\mathrm{P}=0.012)$, control cells cultured for 10 days $(\mathrm{P}=0.003)$, and control cells cultured for 21 days without passages $(\mathrm{P}=0.001)$.

Immunofluorescence staining of the differentiated HUCMSCs revealed the expression of Albumin in 3D culture conditions (Figure. 7). The results showed an increase in the intensity of the reactions to albumin in the cells cultured within a 3D collagen scaffold for long-term (21 days) compared to those cultured for a short period of time (10 days). Albumin was detected in the cells which were exposed to hepatogenic media with or without FGF4. No changes were detected in the comparison of different groups.

\section{Functional Assay}

The glycogen storage capacity of the UCMSCs was evaluated by PAS staining. The non-treated UCMSCs which were cultured for

Figure 3. Alizarin red/S staining revealed $\mathrm{Ca}+$ deposition in the cells cultured in osteogenic medium (a) and its absence in the control cultures (b). Oil red $O$ revealed the presence of lipid droplets in the cells cultured in adipogenic medium (c) and its absence in the control cultures (d).

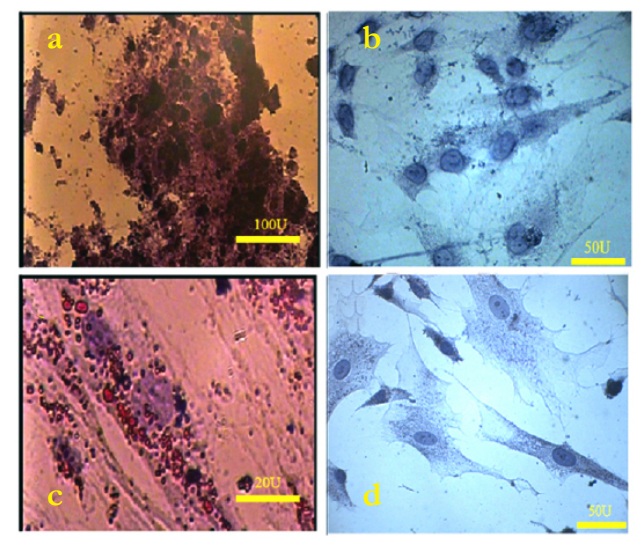


Figure 4. The inverted microscopy of the cells cultured in 3D collagen scaffold showed that the cell morphology was changed in the cultures exposed to hepatogenic media with (a) or without FGF4 (b) compared to the control culture (c).

The nucleus was counterstained with hematoxylin.
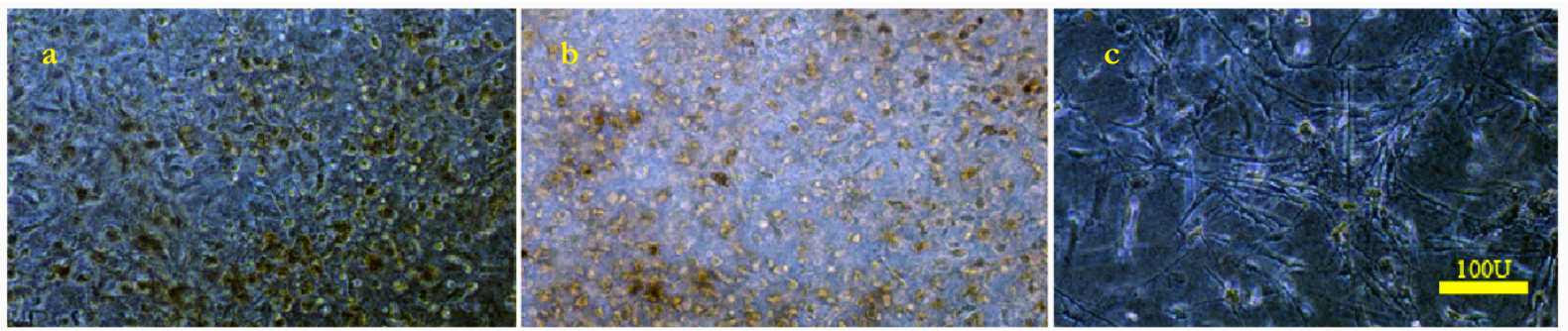

Figure 5. Real time analysis of the expression level of liver-specific markers in HUCMSCs cultured in DMEM (control) in the hepatogenic media with or without FGF4. The cells exposed to FGF4 showed a higher expression level of liver-specific genes, except for claudin and G6P compared to the hepatogenic-treated and control cultures.
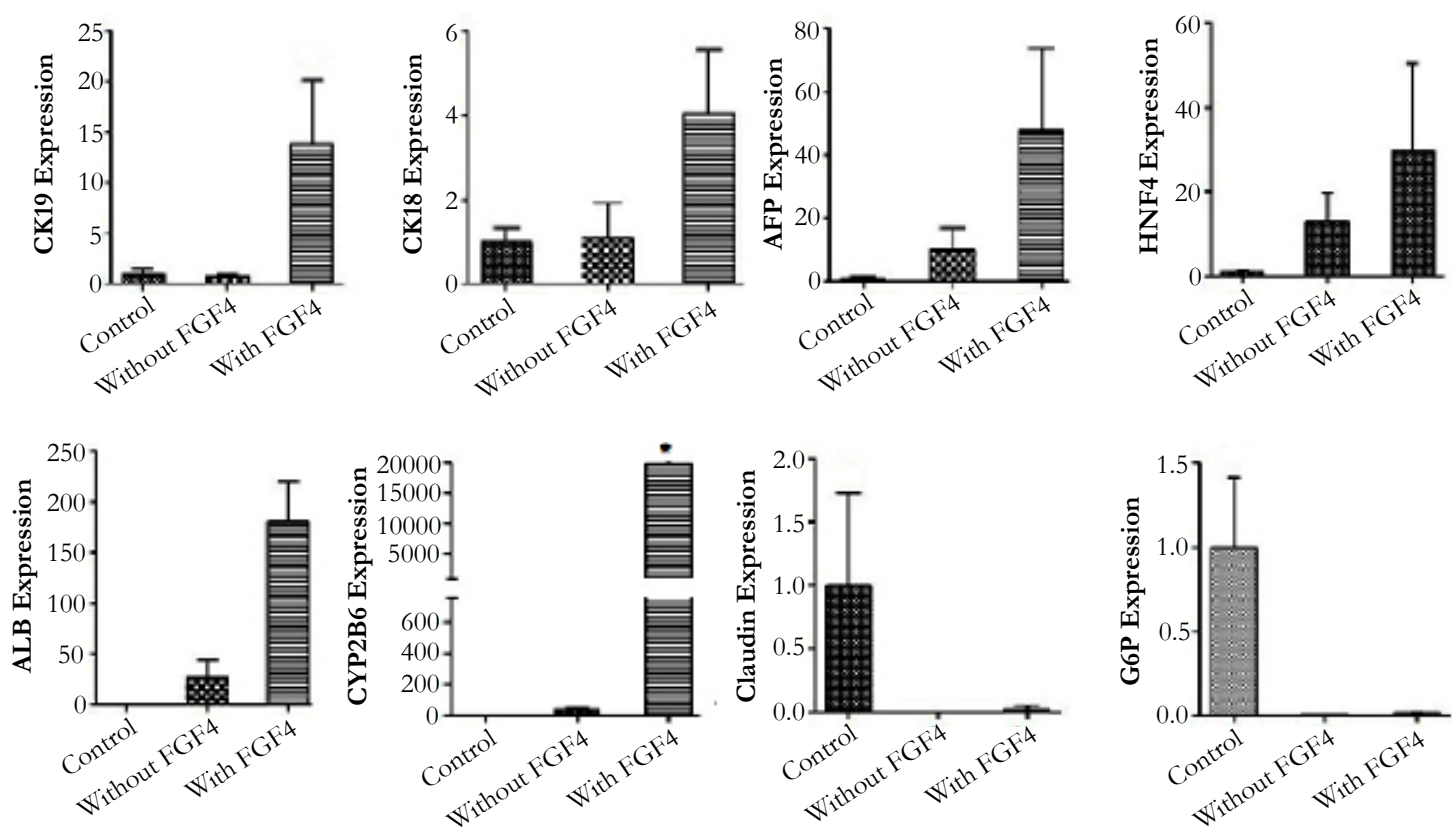

Figure 6. The immunocytochemistry showed low expression of cytokeratin 19, 18 and alpha-fetoprotin in the umbilical cord mesenchymal stem cells cultured for 10 days without passaging (a-c). Long-term culturing the cells led the cells to express cytokeratin 19, 18 and alpha-fetoprotein at a higher level; however, the cells showed a fibroblast-like morphology (d-f). The cells exposed to the hepatogenic media with or without FGF4 also expressed a high level of this protein (g-1). Some cells exposed to hepatogenic media came together and formed a tubular structure (k). These cells also were positive for cytokeratin 19. The rest of the cells revealed round shape morphology after treatment with hepatogenic media (g-l).

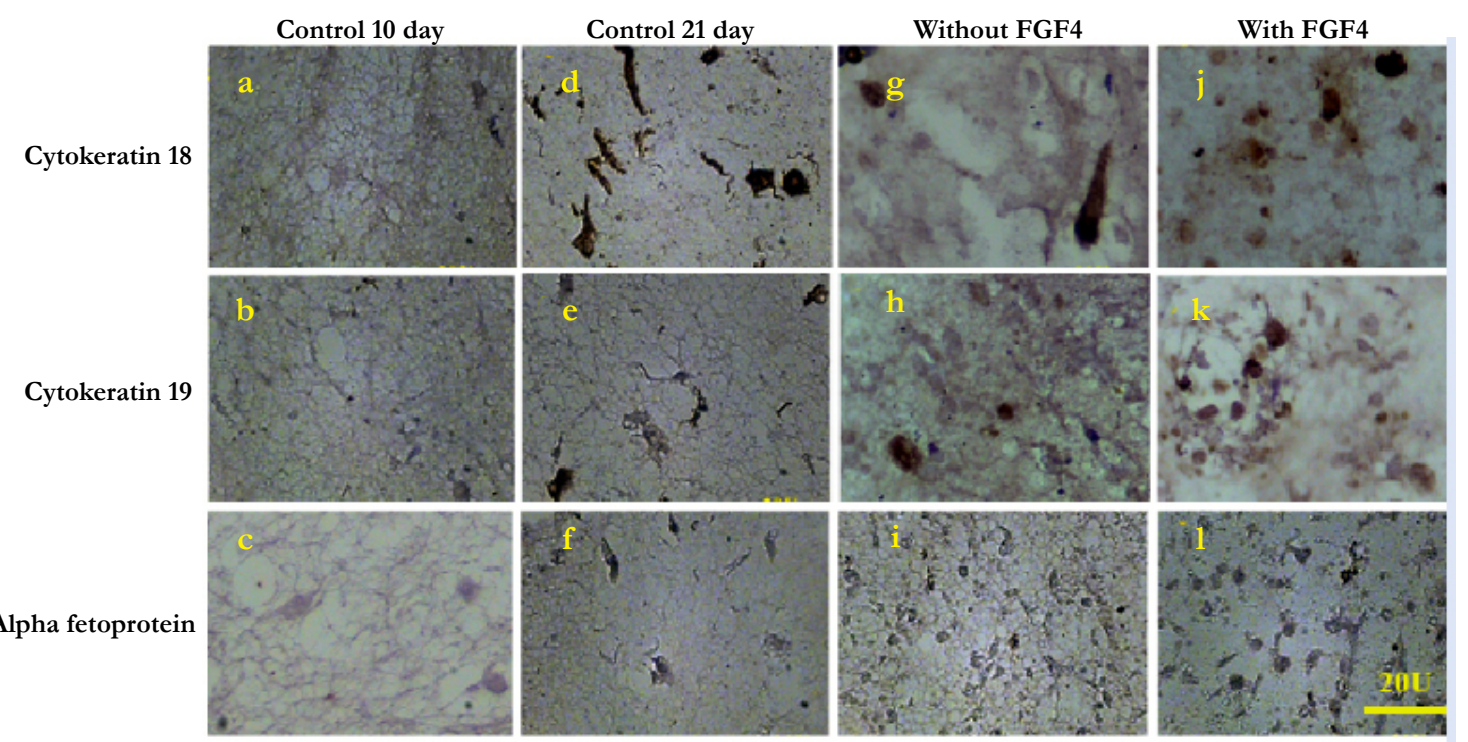


Table 2. the mean of the percent and standard deviation of the reacted-cells to the cytokeratin 18,19 and alpha fetoprotein antibodies.

\begin{tabular}{|c|c|c|c|c|}
\hline $\begin{array}{c}\text { Liver specific } \\
\text { markers }\end{array}$ & $\begin{array}{c}\text { Control (21 days) } \\
\text { (mean } \pm \text { SD) }\end{array}$ & $\begin{array}{c}\text { Control (10 days) } \\
\text { (mean } \pm \text { SD) }\end{array}$ & $\begin{array}{c}\text { Without FGF4 } \\
\text { (mean } \pm \text { SD) }\end{array}$ & $\begin{array}{c}\text { With FGF4 } \\
\text { (mean } \pm \text { SD) }\end{array}$ \\
\hline Cytokeratin 18 & $34.34 \pm 5.19$ & $42.15 \pm 1.36$ & $48.36 \pm 5.86$ & $69.11 \pm 0.54^{*}$ \\
\hline Cytokeratin 19 & $30.63 \pm 3.32$ & $35.7 \pm 7.07$ & $71.76 \pm 3.88^{*}$ & $63.96 \pm 1.95^{*}$ \\
\hline Alpha-fetoprotein & $40.50 \pm 1.06 £$ & $23.50 \pm 3.99$ & $60.01 \pm 4.67 ¥$ & $64.80 \pm 4.45 ¥$ \\
\hline
\end{tabular}

$*$ Significant difference with all other groups $(\mathrm{P}<0.05)$

$¥$ Significant difference with two control groups $(\mathrm{P}<0.05)$

E. Significant difference with control (10 days) $(\mathrm{P}<0.05)$

Figure 7. Immunofluorescence micrographs showed that HUCMSCs expressed albumin in the presence or absence of hepatogenic media in 3D culture scaffold and pre-treated cultures with or without FGF4. There was no change between groups.
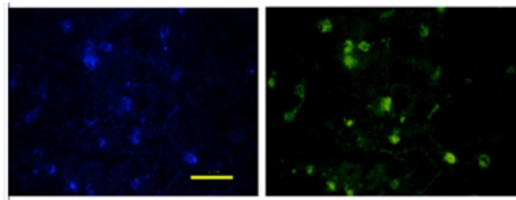

Control 10 day
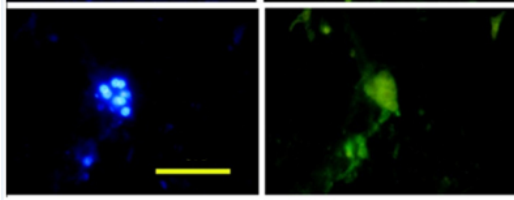

Control 21 day
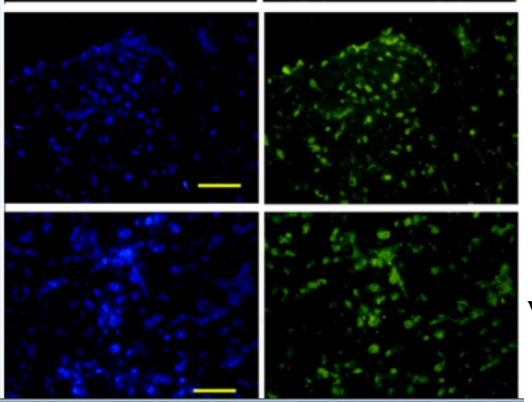

With FGF4

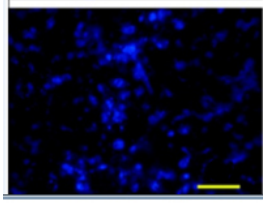

Without FGF4

10 days without passage showed a low level of glycogen storage (24\%). However, long-term cultured cells (for 21 days) $(70 \%)$ demonstrated the ability to store glycogen. The hepatogenic media with $(78 \%)$ or without $(73 \%)$ FGF4 also induced the UCMSCs to show a "strong" positive reaction to PAS staining (Figure 8).

\section{Indocyanine Green (ICG) Uptake}

The cellular uptake and release of ICG were assessed in the differentiated and undifferentiated cells in 3D culture systems on day 21. The statistical analyses showed a significant increase in the ICG released by the cells incubated in hepatogenic media with or without FGF4 $(\mathrm{P}=0.029)$ compared to the control group. However, no significant difference was observed in ICG elimination after supplementation of the culture medium with FGF4 compared to the hepatogenic-treated cells without FGF4. Furthermore, keeping the cells in 3D collagen scaffold for long term led to a non-significant increase in ICG elimination in comparison to the controls (Figure 9).

\section{Discussion}

Human UCMSCs showed a hepatogenic potential and could consequently be used as an important source in treatment of many liver diseases. Moreover, they could express a combination of several early and mid-hepatic markers, such as AFP, CK-18, and
CK-19. Our findings showed a low level of liver-specific mRNA expression in the UCMSCs cultured for long-term without any hepatogenic supplementation, which was in agreement with the findings of a previous research [8]. The results of immunohistochemistry showed that the intensity of the reactions to liverspecific markers increased after long-term culturing in 3D collagen scaffold. Thus, it can be suggested that the cells release some cytokines which are responsible for accelerating the expression of liver-specific markers [8]. This growth factor can induce the cells to express liver-specific markers, such as albumin and CKs $[8,12]$. Despite the expression of liver-specific markers, no change was observed in the cell morphology in the absence of hepatogenic media. These results, along with the findings of other researchers, showed that these cells had a native potential to express liverspecific genes and might be one of the best cell source for differentiation into hepatocytes $[3,8]$.

The supplementation of the culture media with hepatogenic factors containing HGF induces the cells to change their morphology. Moreover, hepatocyte growth factor induces the signaling pathway which is responsible for cell proliferation and organ morphogenesis during liver development [19]. Hepatogenic medium was shown to induce human umbilical cord blood MSCs to change into the small, round, and epithelioid shape [14]. The density of the cells in $3 \mathrm{D}$ cultures also increased in the presence of hepatogenic media. Both HGF and FGF4 exert a mitogenic 
Figure 8. The PAS assay showed that the umbilical cord mesenchymal stem cells cultured for 10 days without passaging could not store a large amount of glycogen (a). Long-term culturing the cells led to an increase in glycogen synthesis; however, the cells showed a fibroblast-like morphology (b). The cells exposed to the hepatogenic media with or without FGF4 also reacted to PAS staining "strongly" (c and d). Some cells exposed to hepatogenic media with FGF4 come together and formed a tubular structure (c). These cells also stored glycogen. The rest of the cells revealed round shape morphology after treatment with hepatogenic media. The cells density was higher in the growth factor-treated cultures.
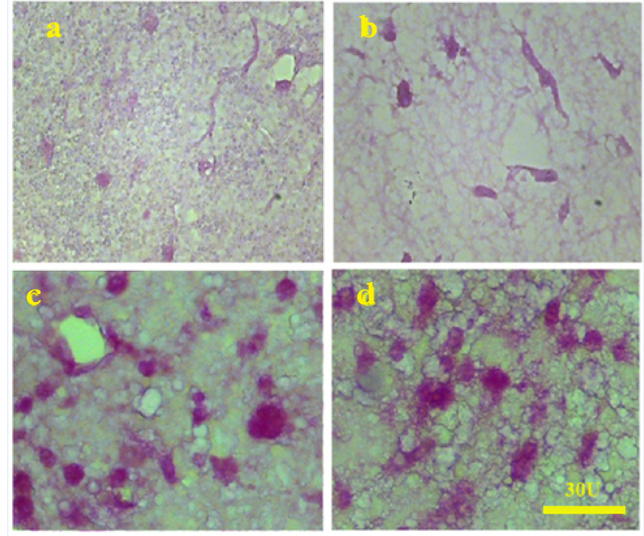

Figure 9. The comparison of the optical density of indocyanine green released from umbilical cord mesenchymal stem cells cultured in DMEM (controls) in the presence of hepatogenic media with or without FGF4 for 2 and 21 days.

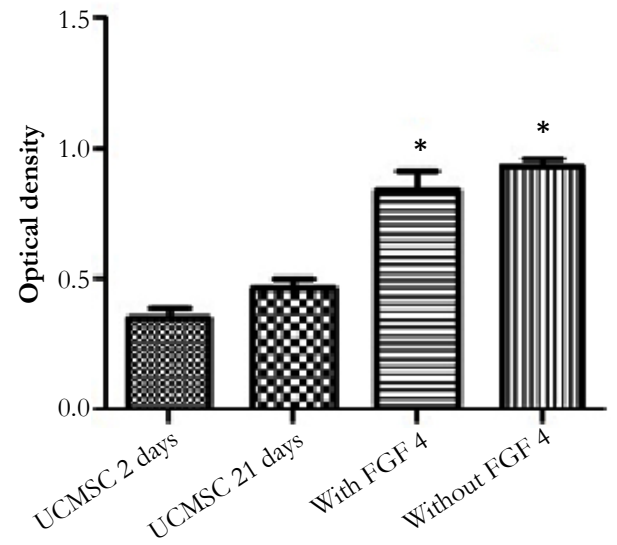

Significant difference with the UCMSC Cultured for 2 days, $(\mathrm{P}<0.05)$

activity which might be the reason for the higher cell density observed in the cultures treated with hepatogenic media [15].

IGF-1 has been shown to induce Embryonic Stem Cells (ESC) to differentiate toward hepatogenic lineage [22]. IGF-I also plays a role in liver development and regeneration and it has been demonstrated to be released in the damaged liver. In addition, it can exert a mitogenic activity and stimulate the secretion of HGF [19] as well as albumin [23]. A combination of IGF-1with HGF, dexamethasone, and OSM were used to induce adipose-derived MSCs toward hepatogenic lineage [19]. FGF4 is a cytokine which induces cell differentiation toward definitive endoderm in vitro. FGF4 has been used in hepatogenic protocols for differentiation of various stem cells, such as human MSCs derived from cord blood [14], and human UCMSCs [18], in 2D culture condition. It has been demonstrated that a combination of FGF4 and HGF could induce bone marrow-derived MSCs into functional hepatocyte-like cells [14]. The data from the present study revealed G6P gene was expressed at a non-significant higher level in the control cultured in 3D collagen scaffold compared with the cells exposed to hepatogenic media. A recent study showed that the adult hepatocytes cultured in the medium containing insulin and Dexa express a lower level G6P. The insulin exerted this inhibitory effect by acting on response sequence (IRS) in the promoter region of the Glc-6-Pase gene [24]. The mRNA expression of endoder$\mathrm{mal}$ and hepatocyte-specific genes was evaluated at varying stages of human ESC differentiation. The differentiated human ESCs demonstrated typical ultrastructural features of hepatocytes [25]. A 29.6 - fold increase in the expression of $\mathrm{HNF}-4 \alpha$, as the main hepatocyte gene, was showed in the cell differentiated into hepatocyte-like cells; however, it was non-significant. The investigations demonstrated that FGFs can induce the HESCs to achieve the ability of HNF-4 $\alpha$ expression [26]. The results demonstrated that both FGF4 and 3D collagen scaffold can be useful for increasing the expression level of mid and late hepatogenic factors such as albumin, AFP, CK18, CK19, CYP2B6 and HNF4- $\alpha$. Besides, real time RT-PCR showed that naive UCMSCs expressed a non-significant high level of claudin when cultured in 3D collagen scaffold. In fact, the expression of claudin is not limited to hepatocytes. Any mesenchymal-epithelial transition is accompanied by an increase in claudin expression [27]. Mesenchymal-epithelial transition may occur in non-treated UCMSCs and may lead to the expression of the tight junction specific marker, claudin. For instance, MSCs secrete Vascular Endothelial Growth Factor which may induce the cells toward endothelial cell lineage through an autocrine signaling pathway [9]. In the present study, the functional assays also showed that all the cultures treated with or without FGF4 could store glycogen and eliminate ICG. The same amount of ICG uptake and elimination was detected in both experimental cultures; however, they released a significant higher concentration of ICG compared to control cultures. 
One study assessed the efficiency of nanofibers collagen scaffold prepared by electrospun on hepatogenic potential of the human bone marrow derived-MSCs and the results indicated a higher liver-specific marker expression [28]. 3D culture in collagen scaffold led to an increase in expression of liver specific markers compared to $2 \mathrm{D}$ culture condition. $3 \mathrm{D}$ culture condition also improved the hepatocyte phenotype [29]. This study also showed that some cells were arranged in a tubular shape, while some others were scattered throughout the scaffold in 3D culture condition. The tubular structure showed a positive reaction to CK19. It has also been demonstrated that CK19 was expressed by bile duct epithelial cells [30].

\section{Conclusion}

In conclusion long-term culturing of UCMSCs in 3D environment could induce the cells to express a higher level of hepatogenic markers at protein level and also they became functional, as well. Therefore, it seems that UCMSCs showed a tendency to differentiate into hepatocyte-like cells after culturing for a long period of time without passaging. Moreover, pre-treatment of the cells with FGF4 before exposure to hepatogenic medium containing IGF-1 and HGF could improve the expression of some late liver-specific markers in mRNA level such as CYP2B6.

\section{Acknowledgments}

The authors would like to thank the Vice-Chancellor of Research in Shiraz University of Medical Sciences for financially supporting the study [grant No. 6205]. They are also grateful for M Salmannejad, M Sani, and E Noori at laboratory for stem cell research, T Heidari at the transplantation research center, and E Nademi at the morphometry and stereology research center for their technical support. The present study was done in fulfillment of the requirements for $\mathrm{PhD}$ degree awarded to $\mathrm{Z}$. Khodabandeh. The authors declare that there is no conflict of interest.

\section{References}

[1]. Garzón I, Pérez-Köhler B, Garrido-Gómez J, Carriel V, Nieto-Aguilar R, et al. (2012) Evaluation of the cell viability of human Wharton's jelly stem cells for use in cell therapy. Tissue Eng Part C Methods 18(6): 408-419.

[2]. La Rocca G, Lo Iacono M, Corsello T, Corrao S, Farina F, et al. (2013) Human Wharton's jelly mesenchymal stem cells maintain the expression of key immunomodulatory molecules when subjected to osteogenic, adipogenic and chondrogenic differentiation in vitro: new perspectives for cellular therapy. Curr Stem Cell Res Ther 8(1): 100-113.

[3]. Nekanti U, Mohanty L, Venugopal P, Balasubramanian S, Totey S, et al. (2010) Optimization and scale-up of Wharton's jelly-derived mesenchymal stem cells for clinical applications. Stem Cell Res 5(3): 244-254.

[4]. Bongso A, Fong C Y, Gauthaman K (2008) Taking stem cells to the clinic: major challenges. Journal of cellular biochemistry. J Cell Biochem 105(6): 1352-1360.

[5]. Ishii T, Yasuchika K, Machimoto T, Kamo N, Komori J, et al. (2007) Transplantation of Embryonic Stem Cell-Derived Endodermal Cells into Mice with Induced Lethal Liver Damage. Stem Cells 25(12): 3252-3260.

[6]. Scheers I, Lombard C, Najimi M, Sokal E (2011) Cell therapy for the treatment of metabolic liver disease: an update on the umbilical cord derived stem cells candidates. Open Tissue Eng Regen Med J 4: 48-53.

[7]. Schmelzer E, Wauthier E, Reid LM (2006) The phenotypes of pluripotent human hepatic progenitors. Stem Cells 24(8): 1852-1858.
[8]. Campard D, Lysy PA, Najimi M, Sokal EM (2008) Native umbilical cord matrix stem cells express hepatic markers and differentiate into hepatocytelike cells. Gastroenterology 134(3): 833-848.

[9]. Miki T, Marongiu F, Ellis EC, Dorko K, Mitamura K, et al. (2009) Production of hepatocyte-like cells from human amnion. Methods Mol Biol 481: 155-168.

[10]. Soto-Gutiérrez A, Navarro-Álvarez N, Zhao D, Rivas-Carrillo JD, Lebkowski J, et al. ( 2007) Differentiation of mouse embryonic stem cells to hepatocyte-like cells by co-culture with human liver nonparenchymal cell lines. Nat Protoc 2(2): 347-356.

[11]. Oh SH, Miyazaki M, Kouchi H, Inoue Y, Sakaguchi M, et al. (2000) Hepatocyte growth factor induces differentiation of adult rat bone marrow cells into a hepatocyte lineage in vitro. Biochem Biophys Res Commun 279(2): 500-504.

[12]. Kakinuma S, Tanaka Y, Chinzei R, Watanabe M, Shimizu-saito K, et al. (2003) Human umbilical cord blood as a source of transplantable hepatic progenitor cells. Stem Cells 21(2): 217-227.

[13]. Kang XQ, Zang WJ, Bao LJ, Li DL, Song TS, et al. ( 2005) Fibroblast growth factor-4 and hepatocyte growth factor induce differentiation of human umbilical cord blood-derived mesenchymal stem cells into hepatocytes. World J Gastroenterol. 11(47): 7461-7465

[14]. Kang XQ, Zang WJ, Song TS, Xu XL, Yu XJ, et al. (2005) Rat bone marrow mesenchymal stem cells differentiate into hepatocytes in vitro. World J Gastroenterol 11(22): 3479-3484.

[15]. Dong XJ, Zhang H, Pan RL, Xiang LX, Shao JZ (2010) Identification of cytokines involved in hepatic differentiation of mBM-MSCs under liver-injury conditions. World J Gastroenterol 16(26): 3267-3278.

[16]. Kasim A, Hayaty N, Govindasamy V, Gnanasegaran N, Musa S, et al. (2012) Unique molecular signatures influencing the biological function and fate of post-natal stem cells isolated from different sources. J Tissue Eng Regen Med. DOI: 10.1002/term.1663.

[17]. Yamada K M, Cukierman E ( 2007) Modeling tissue morphogenesis and cancer in 3D. Cell 130(4): 601-610.

[18]. Yoon HH, Jung BY, Seo YK, Song KY, Park JK (2010) In vitro hepatic differentiation of umbilical cord-derived mesenchymal stem cell. Process Biochem 45(12): 1857-1864.

[19]. Ayatollahi M, Soleimani M, Geramizadeh B, Imanieh M H (2011) Insulinlike growth factor 1 (IGF-I) improves hepatic differentiation of human bone marrow-derived mesenchymal stem cells. Cell Biol Int 35(11): 1169-1176.

[20]. Ye J, Coulouris G, Zaretskaya I, Cutcutache I, Rozen S, et al. (2012) PrimerBLAST: a tool to design target-specific primers for polymerase chain reaction. BMC bioinformatics 13(1): 134.

[21]. Ho CM, Dhawan A, Hughes RD, Lehec SC, Puppi J, et al. (2012) Use of indocyanine green for functional assessment of human hepatocytes for transplantation. Asian J Surg 35(1): 9-15.

[22]. Magner NL, Jung Y, Wu J, Nolta JA, Zern MA, et al. (2013) Insulin and IGFs enhance hepatocyte differentiation from human embryonic stem cells via the PI3K/AKT pathway. Stem Cells 31(10): 2095-2103.

[23]. Tutau F, Rodríguez-Ortigosa C, Puche JE, Juanarena N, Monreal I, et al. (2009) Enhanced actions of insulin-like growth factor-I and interferon- $\alpha$ co-administration in experimental cirrhosis. Liver Int 29(1): 37-46.

[24]. Streeper RS, Svitek CA, Chapman S, Greenbaum LE, Taub R, et al. (1997) A multicomponent insulin response sequence mediates a strong repression of mouse glucose-6-phosphatase gene transcription by insulin. J Biol Chem 272(18): 11698-11701.

[25]. Hamazaki T, Iiboshi Y, Oka M, Papst PJ, Meacham AM, et al. (2001) Hepatic maturation in differentiating embryonic stem cells in vitro. FEBS Lett 497(1): 15-19.

[26]. Ameri J (2010) FGF signaling in specification of hESC-derived definitive endoderm. In Lund University, Faculty of Medicine Doctoral Dissertation Series 2010(6).

[27]. Turksen K (2011) Claudins and cancer stem cells. Stem Cell Rev Rep 7(4): 797-798.

[28]. Ghaedi M, Soleimani M, Shabani I, Duan Y, Lotfi AS (2012) Hepatic differentiation from human mesenchymal stem cells on a novel nanofiber scaffold. Cell Mol Biol Lett 17(1): 89-106.

[29]. Baharvand H, Hashemi SM, Ashtiani SK, Farrokhi A (2006) Differentiation of human embryonic stem cells into hepatocytes in $2 \mathrm{D}$ and $3 \mathrm{D}$ culture systems in vitro. Int J Dev Biol 50(7): 645-652.

[30]. Stosiek P, Kasper M, Karsten U (1990) Expression of cytokeratin 19 during human liver organogenesis. Liver 10(1): 59-63. 